\title{
Albrecht von Haller und Anton de Haen im Streit um die Lehre von der Sensibilität
}

\author{
Von ERNa Lesky
}

Es ist eine Beobachtung, die man in der Historiographie häufig machen kann, daß sie auf bestimmte Ereignisse und Beziehungen im Leben eines Forschers oder auf bestimmte Aspekte seiner Lehre alles Licht zu versammeln pflegt, während andere nicht weniger für Persönlichkeit, Werk und Zeitmilieu typische Gegebenheiten im Schatten zu bleiben verurteilt sind. Auch in der dem großen Schweizer Forscher gewidmeten bio- und ergographischen Literatur verhält es sich zum Teil so. In ihr stehen seine Beziehungen zu Zeitgenossen wie La Mettrie, Voltaire, Linné, Hamberger u. a. seit jeher im Vordergrund des Interesses und haben dementsprechend wiederholte und einläßliche Behandlung erfahren ${ }^{1}$. Anderseits scheint es seinem nicht weniger aufschlußreichen und bewegten Verhältnis zu seinen ehemaligen Leidener Kommilitonen Gerard van Swieten (1700-1772) und Anton de Haen (1704-1776), den späteren Häuptern der Wiener Schule, beschieden zu sein, nur am Rande vermerkt zu werden. So muß man, um einigermaßen Anhaltspunkte für die Darstellung der Beziehungen Hallers zu van Swieten zu bekommen ${ }^{2}$, bis zu Johann Georg Zimmermanns Biographie von $1755^{3}$ zurückgehen. Bei Hallers Auseinandersetzung mit de Haen, deren Darstellung sich diese Untersuchung zur Aufgabe setzt, ist das nicht ganz so schlimm. Da kann man bereits 1828 bei KurT SpRENGEL $^{4}$ eine ganze Seite finden und 1839 bei J. F. C. Hecker ${ }^{5}$ sogar über zwei. Und noch H. HAESER ${ }^{6}$ bringt 1881 darüber etwas über dem Strich, bevor es endgültig Usus wird, diese Angelegenheit unter den Strich zu ver-

1 Vgl. die Angaben bei StePHen D'IrSAY, Albrecht von Haller, Arbeiten aus dem Institut für Geschichte der Medizin an der Universität Leipzig, Band 1, Leipzig 1930, S. 87 ff. Die vorliegende Untersuchung wurde durch die stete Hilfsbereitschaft der Universitätsbibliothek Wien und der Bibliothek der Gesellschaft der Ärzte zu Wien ermöglicht. Dafür sei an dieser Stelle herzlicher Dank gesagt.

2 Vgl. Gesnerus 15 (1958) 120.

3 Johann Georg Zimmermann, Das Leben des Herrn von Haller, Zürich 1755.

${ }^{4}$ K. Sprengel, Versuch einer pragmatischen Geschichte der Arzneykunde, 5. Teil, Halle 1828, S. $415 \mathrm{f}$.

${ }^{5}$ J.F.C.Hecker, Geschichte der neueren Heilkunde, Berlin 1839, S. 4.15 ff.

${ }^{6}$ H. HAESER, Lehrbuch der Geschichte der Medicin und der epidemischen Krankheiten, 2. Band, Jena 1881, S. 581. 
weisen. Dabei stimmen alle Autoren überein, daß gerade dieser Auseinandersetzung um die Hallersche Lehre besondere Bedeutung zukäme, da hier der größte Kliniker seiner Zeit, Anton de Haen in Wien, vom praktischen Standpunkt aus seine Bedenken gegen den Theoretiker anmelde. So darf man von einer Beschäftigung mit dieser Auseinandersetzung wohl erwarten, daß sie, wenn auch die Lehre Hallers nicht in neuem, so doch in viel schärferem Licht hervortreten lasse.

Die Irritabilitätslehre ${ }^{6}$ a wird zwar in jeder Haller-Würdigung als die epochale Leistung mit der Feststellung gewertet, daß es erstmals gelang, spezifische, vitale Funktionen wie Sensibilität und Irritabilität auf Grund des systematisch angewendeten Experiments bestimmten Strukturen des Gewebes, dem Nerv bzw. dem Muskel, zuzuordnen. An diese Feststellung schließen sich meist kritische Bemerkungen an über die Enge des Hallerschen Irritabilitätsbegriffes (Irritabilität $=$ Kontraktilität) und über die Korrekturen und Generalisierungen, die er in der Folge besonders in den Schulen von Montpellier und Edinburgh erfuhr. Damit ist sicherlich die historische Bedeutung sowie die Entwicklungsrichtung der Hallerschen Lehre bezeichnet, aber in der Tat sehr wenig ausgesagt über die Fülle von speziellen Problemen, die diese Lehre für die zeitgenössische Medizin in sich barg. Im besondern scheint jener Teil von ihr zu kurz zu kommen, der sich mit der Klärung der Sensibilitätsverhältnisse befaßt. Aber gerade dieser Teil war es, der dem Praktikus am Krankenbett die größten Schwierigkeiten bereitete. Diese an einigen signifikanten Beispielen aus der de Haenschen Auseinandersetzung zu beleuchten, dürfte nicht nur die Feinstruktur der Sensibilitätslehre Hallers besser kennen lehren, sondern auch einige Probleme der zeitgenössisch-klinischen Medizin schärfer erfassen lassen. Bevor wir jedoch auf diese eingehen, sei zuerst einiges über die Lehre selbst ausgesagt und über den Boden, den sie in Wien vorfand.

\section{Ein neues System}

Wir dürfen es de Haen glauben, auch wenn er es nicht so oft ausgesprochen hätte, daß es die Angst vor einem neuen System und seinen Auswirkungen auf die praktische Medizin war, die ihn die Feder gegen Haller er-

6a Vgl. die umfassenden Darstellungen bei H.Buess, Zur Entwicklung der Irritabilitätslehre, Festschrift Brodbeck-Sandreuter, Basel 1942, S.299-333; K. E. RотнschUн, Vom Spiritus animalis zum Nervenaktionsstrom, in Ciba-Zeitschrift Nr.89, Band 8, Wehr/ Baden 1958. 
greifen ließ. Schließlich hatte das vergangene Jahrhundert und zum Teil de Haen selbst genug solcher Systeme emporwachsen und noch schneller dahinsinken sehen. In seiner Lettre à un de ses amis ${ }^{7}$ erinnert daher de Haen den Verteidiger von Hallers Lehre, Samuel Auguste André David Tissot (1728-1797) in Lausanne, an die Systeme der Helmontianer, Cartesianer, an jenes des Bontekoe, des Craanen, de le Boe-Sylvius und auch an das Stahls, wie sie alle nur erschienen waren, um eines dem andern Platz zu machen, nachdem sie viel Verwirrung und wenig Nutzen in der Heilkunde gestiftet hätten. Dasselbe befürchtete de Haen auch von der neuen Lehre Hallers, die er über die Sensibilität und Irritabilität der Körperteile am 22. April und 6. Mai 1752 in der königlichen Gesellschaft der Wissenschaften zu Göttingen ${ }^{8}$ vorgetragen hatte. Sicherlich hatte er dabei erwiesen, daß nur der Nerv empfinden, nur die Muskelfaser sich zusammenziehen, d.h. reizbar sein könne. Aber er hatte gleichzeitig auch versucht, diesen als fundamental erkannten Eigenschaften alle Körperteile unterzuordnen. An der Richtigkeit dieser Zuordnung zweifelte de Haen, besonders aber daran, daß Sehnen, Bänder, Gelenkkapseln, Periost, Dura und Pia Mater und sogar Rippen- und Bauchfell gänzlich unempfindlich sein sollten, weil sie keine Nerven besäßen, andere hinwiederum, wie Lunge, Leber, Milz, Nieren, nur stumpf empfänden, da sie wenig Nerven aufwiesen. Und ähnlich waren Herz, Zwerchfell und auch der Darm zufolge ihrer muskulösen Struktur als außerordentlich reizbare Teile deklariert, dagegen als kaum reizbare Lunge, Leber und Nieren wegen ihrer zellichten Textur.

Bei Haller waren dies nur experimentell erhobene Befunde und sollten auch nicht mehr sein. Stets hat sich der Meister des Experiments und noch im achten Band der Elementa ${ }^{9}$ dagegen gewehrt, « daß man des Hallerschen Systems Erwehnung thut, da ich doch blos gesagt, daß diejenigen Theile

7 A. De Haen, Lettre de Mr. de Haen à un de ses amis au sujet de la lettre de Mr. Tissot à Mr. Hirzel, Vienne 1763, S. 83. Dazu vgl. H. W. Bucher, Tissot und sein traité des nerfs, Zürcher Medizingeschichtliche Abhandlungen, N. R. 1, Zürich 1958, S. $10 \mathrm{ff}$.

8 Albertus de Haller, De partibus corporis humani sensilibus et irritabilibus, Comment. Soc. Reg. Gotting., tom. II ad annum 1752, Gotting. 1753, wird hier zitiert nach der Ausgabe K. Sudhoffs: Albrecht von Haller, Von den empfindlichen und reizbaren Teilen des menschlichen Körpers, «Klassiker der Medizin», Band 27, Leipzig 1922.

${ }^{9}$ Hallers Elementa physiologiae corporis humani, Lausanne 1757-1766, werden im folgenden nach der deutschen Übersetzung Johann Samuel Hallens, Herrn Albrecht von Hallers Anfangsgründe der Phisiologie des menschlichen Körpers, Berlin und Leipzig 1759-1776, als «Elementa» zitiert. Zu ihrer Entstehung vgl. jetzt H. BuEss, Gesnerus 15 (1958), 17-35. 
empfinden oder sich bewegen, die ich sie empfinden oder bewegen sahe: so wie ich diejenige für unempfindlich ausgegeben, denen ich weder Gefühl noch eine angebohrne Bewegung wahrgenommen ». Aber trotz dieses Protestes hat Haller es nicht verhindern können, daß bereits seine Zeitgenossen aus seinen Befunden Systeme machten. Nicht erst in Montpellier und Edinburgh wurde dieser Schritt getan, sondern bereits $1760 \mathrm{im}$ Discours préliminaire zu den Mèmoires ${ }^{10}$. Sein Freund und Verteidiger, der nachmalige Kliniker von Pavia, S.A.A.D.Tissot war es, der die parties sensibles et insensibles sowie die parties irritables et airritables nicht nur in ein Schema, in eine table, zwang, sondern über diese ein allgemeines Sensibilitätsgesetz schrieb derart, daß härtere und kompaktere Körperteile weniger sensibel seien als solche von weichem und lockerem Bau. Es war für de Haen ein Leichtes, Tissot mit der ganz simplen Frage zu widerlegen ${ }^{11}$, ob die Vertreter dieser Lehre niemals an Zahnweh gelitten hätten. Aber nicht nur dieses Sensibilitätsgesetz konnte de Haen in der Vorrede Tissots bereits formuliert finden; in ihr wurden auch schon die Grundzüge eines pathologischen Systems mit all seinen therapeutischen Konsequenzen, besonders in den Ausführungen über die Opiumwirkung und das Fieber ${ }^{12}$, sichtbar, zu denen die Generalisierung des Hallerschen Irritabilitätsbegriffes zum principe vital unweigerlich führen mußte und tatsächlich in den Systemen Cullens und Browns auch geführt hat.

So war die Verlockung zu System- und Hypothesenbildung und auch schon die wirklichen Ansätze dazu von allem Anfang an da. De Haen hat diese systembildende Kraft in der Hallerschen Lehre sogleich gespürt und auch gefürchtet. Denn nirgends war man für Systeme und Hypothesen so sehr empfindlich und diese mehr verhaßt als bei den Boerhaave-Schülern in Wien. Während deren einstiger Kommilitone und spätere Göttinger Professor, Haller, die Hypothese als eine «Nothmünze» ${ }^{13}$, aber doch als ein nützliches Instrument zur Wahrheitsfindung anerkannte, lehnte sie das Wiener Schulhaupt, van Swieten, grundsätzlich ab. «Exulant hypotheses», verkündete er in einer 1759 gehaltenen Rede ${ }^{14}$, die den Wahlspruch seines

10 Alb. de Haller, Mémoires sur la nature sensible et irritable des parties du corps animal, Lausanne 1756-1762. Im folgenden zitiert als «Mém.». Dazu vgl. H. Buess (Anm. 6a), S. $325 \mathrm{f}$.

11 Lettre de Mr. de Haen (vgl. Anmerkung 7), S. 77.

12 Mém. 1, XXI ff.

13 Sammlung kleiner Hallerischer Schriften, 1. Teil, Bern 1772, S. 59.

14 Gerh. L. B. van Swieten, oratio de medicina simplici vera, Viennae, o. J., S. 26. Diese für die Methode der Wiener Schule so aufschlußreiche Rede vaN SwIETENs hat man in der bisherigen Literatur ganz außer Betracht gelassen. 
Lehrers Boerhaave «simplex veri sigillum» für die Wiener Schule zum Programm erhebt. Er weiß sich dabei eins mit seinem Mitarbeiter und Freunde de Haen, der gleich auf den ersten Seiten seines Lebenswerkes, der Ratio medendi ${ }^{15}$, und später in demselben Werke unter ausdrücklicher Bezugnahme auf Haller erklärte ${ }^{16}$, er binde sich an keine Systeme, diese «Pest in der Arzneywissenschaft». Denn in der unvoreingenommenen Beobachtung der Natur, wie Hippokrates und Sydenham sie geübt ${ }^{17}$, sähe er die einzig richtige Methode der Forschung, wenngleich auch sie niemals uns die ersten und wahren Ursachen der Dinge erkennen lasse.

Hier finden wir in der Stellungnahme zu Haller von de Haen bereits jene skeptisch-agnostische Haltung der Wiener Schule bezogen, die nachmals der Initiator der französischen Schule, P.J.G. Cabanis (1757-1808), so lebhaft akklamiert und für diese Schule als nachahmenswertes Prinzip gefordert hat ${ }^{18}$. In diesem Skeptizismus fühlt sich de Haen in genauem Gegensatz zu der Zuversicht, mit der Haller zu Werke geht und über deren Auswirkung er vermerkt: ${ }^{19}$ "Man jauchzet anjetzt in Europa, daß man bey der weitern Aufklärung der Physiologie alle Schwierigkeiten . . . völlig und glücklich gehoben hätte», in Wirklichkeit aber widersprächen sich die Experimente ebenso wie die daraus gezogenen Schlüsse.

Man könnte nun meinen, daß dieser Skeptizismus de Haens gegen die Hallersche Lehre ebenso wie seine und van Swietens Hypothesen- und Systemfeindlichkeit auch anderen, persönlicheren Motiven gegen den Urheber der Irritabilitätslehre entsprängen, an denen es wahrlich nicht gefehlt hat, wie bereits früher ${ }^{20}$ gezeigt wurde. $\mathrm{Da}$ solche Motive jedoch nicht im Vordergrunde standen, vielmehr die Hypothesen- und Systemfeindlichkeit der Wiener Schulgründer eine programmatische war und für die ganze Schule verbindlich, ja letztlich zu einem signifikanten Merkmal eben dieser Schule wurde, macht nichts offenkundiger, als da $\beta$ man nach dem Tode

15 A. DE HAEN, Ratio medendi in nosocomio practico, 15 voll., Vindob. 1756-1773, wird im folgenden nach Teil (=1. Ziffer), Kapitel (= 2. Ziffer) und Seite (= 3. Ziffer) der deutschen Übersetzung von ERnst Platner, Heilungsmethode in dem kaiserlichen Krankenhause zu Wien, 8 Bände, Leipzig 1779-1784, als «Rat. med.» zitiert. Die angeführte Stelle dort $1,1,4$.

16 Rat. med. 9, 4, 298; 9, 2, 229.

17 Rat. med. 8, 3, 110;2, 1, $90 \mathrm{ff}$.

18 P.J.G. Cabanis, Du degré de certitude de la médecine, Paris 1798, S. 70. Dazu vgl. Gesnerus 11 (1954) $155 \mathrm{ff}$.

19 Rat. med. 2, 8, 176.

20 In dem in Anmerkung 2 zitierten Aufsatz. 
van Swietens (1772) 1775 sogar statutenmäßig in der von ANToN voN SтöRcK redigierten Wiener Fakultäts-Einrichtung ${ }^{21}$ verankerte: «Die Hauptsorge in unserer Schule ist es, daß die Schüler selbst . . . nicht durch Hypothesen, die in der Ausübung der Heilkunst meist nur schaden, angesteckt werden ».

Dieses grundsätzlich skeptische und grundsätzlich konservative Wiener Programm muß man also in Rechnung setzen, wenn man Standort und Ausgangspunkt de Haens in seiner Auseinandersetzung mit Haller richtig bestimmen und auch verstehen will. Es war tatsächlich so, wie es de Haen in der Dedicatio seiner ersten 1761 erschienenen Streitschrift, den Difficultates $^{22}$, darstellte und 1768 in der Beendigung der Streitfragen ${ }^{23}$ und 1769 in dem Brief an Haller ${ }^{24}$ wiederholte: Als erster Professor der praktischen Heilkunde in Wien konnte er sich von Amts wegen nicht nur berufen, sondern geradezu verpflichtet fühlen, zu jedem System Stellung zu nehmen. Und Hallers neue Lehre schien ihm nun einmal aus den früher berührten Gründen dazu angetan, das «systema modernorum» schlechtweg zu sein, vor dem seine Schüler zu warnen und zu bewahren, er als sein officium betrachtete.

Man kann gerade nicht sagen, daß dieses officium dem Wiener primarius praxeos sehr schwergefallen wäre. Im Gegenteil. Es war ein sehr streitlustiger Mann, den sich van Swieten 1754 von seiner Praxis im Haag weg auf die Wiener Lehrkanzel geholt hat. de Haens bedeutendster Schüler, jene anima candida der Ärzte Altwiens, Maximilian Stoll (1742-1787), hat nachmals mit Bedauern geradezu von der «lubido litigandi» seines Lehrers gesprochen ${ }^{25}$. Diese mußte nicht nur van Swieten selbst erfahren und mancher prominent Gewordene des Schülerkreises wie ANтon STörck (1731-1803), Joseph Quarin (1734-1814) und Matthäus Collin (1739-1817), die im Schierlingsstreit ${ }^{26}$ mit ihren Anhängern als «Cicutarii » verdammt wurden; über die Gelehrten halb Europas goß sich diese herrschsüchtige Streitbarkeit wegen verschiedener Differenzen aus: bei John Pringle (1707-1782),

${ }^{21}$ Instituta facultatis medicae Vindobonensis cur. Ant. Störck, Vindob. 1775, S. 9.

22 A. DE HAEN, Difficultates circa modernorum systema de sensibilitate et irritabilitate humani corporis, Viennae 1761. Im folgenden zitiert als «Difficult.».

${ }^{23}$ Rat.med. 12, 7, 390 f.

24 Epistolarum ab eruditis viris ad Alb. Hallerum scriptarum pars I. Lat., Vol. VI, Bernae 1775, Nr. 280, S. 16. Im folgenden zitiert als «Epist.».

25 A. DE HaEN, Opuscula quaedam inedita, herausgegeben von J. Eyered, P. I, Vindob. 1795, S. XI f.

${ }^{26}$ Alethophilorum quorundam Viennensium elucidatio necessaria epistolae de cicuta, Vindob. 1766. 
dem Boerhaave-Schüler in London, waren es solche über Petechien und Friesel, bei Théophile de Bordeu (1722-1776) in Paris ging es um Pulsqualitäten, Balthasar Ludwig Tralles (1708-1797) in Breslau und Charles Marie de la Condamine (1701-1774) in Paris meinten es anders in der Inokulationsfrage, und der schon genannte S.A.A.D. Tissot in Lausanne überhaupt in allem: über den Sitz der Pleuritis, über Petechien und Friesel und über die Inokulation ${ }^{27}$. Und jetzt 1762 hatte er auch noch Hallers Lehre von der Irritabilität gegen ihn, de Haen, verteidigt, und dasselbe wagte sogar in der eigenen Fakultät in Wien der Kollege auf dem Lehrstuhl der Materia medica, Heinrich Johann Nepomuk Crantz (1722-1797) ${ }^{28}$, nur in Groningen stand 1765 der Professor der Medizin, Walther vaN Doeveren ${ }^{29}$, zu ihm.

Haller hat schon recht gehabt, wenn er in seiner 1761 erschienenen Apologia $^{30}$ die Rolle de Haens in der Gelehrtenrepublik mit jener in Hobbes' Staat verglich: Einer gegen alle, alle gegen einen waren zum Kampf angetreten, und das bellum literarium herrschte schier schrankenlos. Es kämpften aber nicht nur die Geistesheroen, Haller, de Haen, Tissot, Crantz: Wie das schon so ist, auch die kleinen, namenlos gebliebenen und bleiben wollenden Geisterchen taten mit und verfaßten Episteln und Elucidationes ${ }^{31}$.

Will man dieses vielstimmige bellum literarium zwischen de Haen und Haller einigermaßen überblicken, dann muß man schon dem Rat eines so guten Literaturkenners wie E.G.BALDINGer folgen und darf es nicht bei den als solchen deklarierten Streitschriften bewenden lassen. Man muß auch einen Großteil der Werke Hallers und de Haens heranziehen, vor allem die Elementa des ersten und die Ratio medendi des zweiten, und ebenso ihren Briefwechsel. Aber nicht nur ihren, sondern auch den mit andern Gelehrten. Und man muß auch die Versuche und Gegenversuche der beider-

${ }^{27}$ Vgl. die in Anmerkung 7 zitierte Streitschrift sowie H. W. Bucher, a.a.O.S.12.

${ }^{28}$ HeINr. ЈоH. Nep. Crantz, Solutiones difficultatum circa cordis irritabilitatem, Viennae 1761. Ferner Lettre de Mr. Crantz à Mr. Tissot au sujet de sa dispute avec Mr. de Haen, Vienne 1763.

29 Gualth. van Doeveren, Specimen observationum acad. ad monstrorum historiam, anatomen, pathologiam, et artem obstetriciam, praecipue spectantium, Groning. 1765, Cap. XIV, S. 208-54.

${ }^{30}$ Alb. v. Haller ad viri ill. Antonii de Haen difficultates apologia, Edit. IV. emend., S. 7.

${ }^{31}$ Außer der in Anmerkung 26 zitierten elucidatio vgl. auch die epistola quam de vindiciis Haenii ad Alb. de Haller lippi et tonsores scripserunt, Hannov. 1762. Dazu die Kritik in den Gött.Anz.v.gel.Sach.1 (1764) 26. St., S. $203 \mathrm{ff}$. 
seitigen Schüler studieren und auch die der dänischen, deutschen, französischen und italienischen Forscher. Glücklicherweise liegen diese zum größten Teil gesammelt vor in der dreibändigen Ausgabe Giacinto BartoLOMeo FabBris, Sulla insensibilità ed irritabilità Halleriana opuscoli di vari autori raccolti ${ }^{32}$ ebenso wie in der Gegensammlung Hallers, den vierbändigen Mémoires sur la nature sensible et irritable des parties du corps animal $^{33}$ und dem ersten Band der Opera minora, Lausanne 1763.

Als de Haen 1761 mit seinem ersten Angriff gegen Haller, seinen Difficultates, herauskam, da kannte er weder Fabbris Opuscoli raccolti noch die ersten drei Bände vonHallers Mémoires. Das macht ihm Haller in seiner Apologia 1761 zum Vorwurf. Hierauf entgegnet 1762 de Haen in den 248 Seiten umfassenden Vindiciae difficultatum ${ }^{34}$, die Haller seinerseits 1763 in der Vorrede zum fünften Band der Elementa beantwortet. Darauf bekommt der 1764 erscheinende zwölfte Teil der Ratio medendi ein eigenes Streitkapitel «Vom Hallerischen System » ${ }^{35}$, während sich Haller in seiner Antwort 1766 nur auf einen Teil der Vorrede zum achten Band der Elementa beschränkt. Damit ist das literarische Grundgerüst des Streites bezeichnet, aus dem uns eine verwirrende Fülle von Stimmen über die hitzig umstrittenen Teilfragen der Sensibilitäts- und Irritabilitätslehre entgegentönt. Um aus diesem vielstimmigen Konzert der Meinungen den Part des Wiener Klinikers immer herauszuhören, sei kurz bezeichnet, worum es diesem vor allem ging:

Zum ersten erblickte de Haen in der Annahme von Hallers Lehre einen Abfall von Hippokrates, der ihm nicht nur Vorbild, sondern die ursprüngliche Lehre der Natur schlechtweg war. Er befürchtete und sah es zum Teil schon verwirklicht, daß die hippokratischen Heilvorschriften «von vielen Ärzten so vernachlässigt wurden, daß sie bey Krankheiten ihr ganzes Augenmerk nur auf einen höheren oder geringeren Grad von Reizbarkeit richteten, und die unbeweglichen Grundsätze unseres ersten Lehrers dabey für unnöthig oder wenigstens für unbedeutend hielten ${ }^{36}$. Diese Befürchtung hat sich denn auch durch die Weiterbildung der Hallerschen Lehre in den

32 Bologna 1757-1759. Im folgenden zitiert als «Op. racc.».

33 Vgl. Anmerkung 10.

34 A. DE HAEN, Vindiciae difficultatum circa modernorum systema de sensibilitate et irritabilitate humani corporis contra viri perill.atque cl. Alb. v. Haller ad easdem difficultates apologiam, Viennae 1762.

35 Rat. med. 9, 2, 237-98.

36 Rat. med. 12, 7, 390. 
Systemen von William Cullen (1712-1790) und John Brown (1735-1788) bestätigt und am Jahrhundertende zum vollen Gegensatz zwischen alter Humoral- und neuer Solidarpathologie vertieft. Diese Entwicklung der Hallerschen Lehre ging vor allem von ihrem Irritabilitätsbegriff aus und betraf in erster Linie die interne Medizin. Während sie in dieser erst nach dem Tode de Haens (1776) zur vollen systemmäßigen Entfaltung gelangte, konnte de Haen eine andere Auswirkung der Lehre Hallers, die sich an ihre Sensibilitätsauffassung anschloß und vor allem die Chirurgie betraf, noch selbst und bereits unmittelbar nach Bekanntwerden der Göttinger Versuche erleben: Eine neue Kühnheit drohte bei den Chirurgen einzuziehen. Denn waren nicht so viele Körperteile erwiesenermaßen unempfindlich? Konnte man jetzt nicht ohne weiteres eine Sehnennaht wagen oder eine Entfernung der Dura mater, wenn sich unter ihr Blut oder Eiter angesammelt ${ }^{37}$ Und wenn das Gleiche unter den Aponeurosen und Faszien der Bauchmuskeln der Fall war, so konnte man auch sie ohne Besorgung von Konvulsionen durchtrennen. Diesen und anderen praktischen Folgerungen zu begegnen, war ein so vordringliches Anliegen de Haens, daß er von den 155 Seiten der Difficultates 94 der Bekämpfung der Sensibilitätslehre widmete. Ihr wollen wir nun unsere Aufmerksamkeit zuwenden, indem wir sie an einem zentral umstrittenen Spezialfall exemplifizieren, an der unempfindlich erklärten Achillessehne.

\section{Ist die Achillessehne ${ }^{38}$ unempfindlich?}

Für die Wundärzte der ersten Jahrhunderthälfte war Lorenz Heisters (1683-1758) 1718 erstmals in Nürnberg aufgelegte Chirurgie ${ }^{39}$ das, was für die Leibärzte Boerhaaves (1668-1738) 1709 in Leiden erschienene Aphorismi de cognoscendis et curandis morbis waren ${ }^{40}$ : ihre Bibel. In diesen Bibeln der äußeren und der inneren Heilkunde konnten sie folgende Regeln finden: Die ärgsten Zufälle (saevissima mala) entstehen bei Wunden

37 Vgl. das in Mém. 2, 279 ff. abgedruckte Vorwort G. Vincenzo Petrinis sowie M.A. Caldani, Mém. 3, $483 \mathrm{f}$.

38 Nach J. Hyrte, Onomatologia anatomica, Wien 1880, S. 531 f., sprach der AnatomieProfessor in Löwen, Philipp VerheiJen (1648-1710), 1693 als erster von der «chorda Achillis». Die Bezeichnung «tendo Achillis » stammt von Lorenz Heister (1683-1758), dem führenden deutschen Chirurgen der ersten Jahrhunderthälfte.

39 Wir benutzten die 2. Auflage, Nürnberg 1724. Die einschlägige Stelle dort S. $803 \mathrm{f}$.

${ }^{40}$ Es wurde die in Leiden 1728 erschienene 4. Auflage benutzt. Vgl. dort § 162 ff., S. $36 f$. 
der Sehnen. Besonders wenn nur die eine Hälfte der Sehne durchtrennt sei oder der Wundarzt die freigelegte leicht berühre, habe er die heftigsten Schmerzen, Krämpfe, ja selbst den Tod des Patienten zu erwarten, dasselbe auch bei partiellen Rupturen, dagegen ganz und gar nicht bei totalen. Er müsse daher jede partielle Verletzung oder Ruptur der Sehne durch einen Transversalschnitt in eine totale verwandeln, um den genannten Folgen von vorneherein vorzubeugen.

Solches konnte der französische Chirurgenzögling bei seinem Lehrer am Jardin du roi, René Jacques Croissant de Garengeot (1688-1759) ${ }^{41}$, hören, der österreichische bei dem Wiener Schulhaupt, van Swieten ${ }^{42}$, lesen und der schwedische bei seinem großen chirurgischen Meister OLoF ACREL $^{43}$. Alle europäischen Chirurgen richteten sich darnach und fürchteten daher Sehnenwunden aufs äußerste. Nicht minder aber auch ihre Patienten. Für deren Angst überliefert uns der führende dänische Chirurg Georg Heuermann (1722-1768) ${ }^{44}$ aus H.F.Le Drans Traité des opérations de chirurgie, Paris 1742, ein eindrucksvolles Beispiel: Ein französischer Offizier war an der Hand verletzt und deshalb von Le Dran operiert worden. «Da der Offizier hierauf die bloßen Tendines der Musculi extensores der vier Finger gesehen, so hat er alsbald ausgerufen, daß seine Tendines verletzet wären, worauf er ebenermaßen augenblicklich verstorben.»

Als Haller am 22. April 1752 vor der Göttingischen Gesellschaft der Wissenschaften sein Versuchsergebnis bekanntgab ${ }^{45}$, «es ist also offenbar, daß in der Sehne weder Empfindung noch Bewegung ist», und daran die Folgerung schloß, «wir wollen also unsere Furcht vor den Wunden der Sehnen ablegen, sie mögen gestochen, gebrannt, gehauen oder geschnitten sein », da war er sich vollkommen bewußt, daß er damit nicht nur ein groBes Dogma des Hippokrates ${ }^{46}$ und des Galen ${ }^{47}$, sondern ein ebensolches

41 René Jacques Croissant de Garengeot, Traité des opérations de chirurgie, 2. Auflage, Paris 1731, t. III, c. VII, p. $261 \mathrm{ff}$.

42 G. van Swieten, Commentaria in Hermanni Boerhaave aphorismos de cognoscendis et curandis morbis, 2. Auflage t. I, Lugd.Bat. 1745, § 164, S. $240 \mathrm{ff}$.

43 Olof ACreL, Uetförlig Förklaring Om Friska Sars Egenskaper, Stockholm 1745, S. $261 \mathrm{f}$. Zu O. Acrel vgl. W. Kock, Kungl. Serafimerlasarettet 1752-1952, Stockholm 1952, S. $111 \mathrm{f}$.

44 Georg Heuermann, Abhandlung der vornehmsten chirurgischen Operationen am menschlichen Körper, 1. Band, Kopenhagen und Leipzig 1754, S. 103.

${ }^{45}$ In der in Anmerkung 8 zitierten Ausgabe, S. 19.

46 Vgl. Hippokrates, Epid. V, 76 (= V 248 L.).

47 Method. med. IV, VI (= X 290.403 K.); Ars med. 31 (= I 388 K.). 
seines Lehrers Boerhaave und der gesamten Chirurgie seiner Zeit ${ }^{48}$ gestürzt habe.

Für die ebenso hippokratisch-konservativen wie pietätvoll-gläubigen Boerhaave-Schüler in Wien mußte dies eine besondere Herausforderung bedeuten, da ja Haller nicht nur ihre antiken Vorbilder, ihren verehrten Lehrer Boerhaave, sondern ebenso van Swieten als Vertreter dieses Dogmas genannt hatte. Beider Verteidigung übernimmt de Haen, indem er repliziert $^{49}$ : Als ob Boerhaave und van Swieten nicht eine Sehne von einem Nerven hätten unterscheiden können! Welch eine Unkenntnis schiebe er, Haller, den spectatissimi doctissimique viri zu in der Meinung, sie hätten wie die antiken Ärzte unter dem Wort «Neuron» Nerven, Sehnen und Bänder verstanden. In der Tat hatte man diesen Irrtum lange vor Boerhaave schon überwunden, aber nur im Grob-Anatomischen, nicht auch im Funktionell-Pathologischen. Da lebte ein Teil der antiken Konfusion, gegen die Haller sich wendet ${ }^{50}$, wirklich noch ungebrochen weiter. Indem Boerhaave und van Swieten die Folgen von Sehnen- und Nervenverletzungen identifizierten, waren sie im Banne der antiken Tradition geblieben, ja sie befestigten diese sogar dadurch, daß sie sie morphologisch neu adaptierten mit der Annahme, die Nervenfäserchen aus dem Muskel setzten sich direkt in die Sehne fort ${ }^{51}$.

Diesen alten und neuen Hypothesen, diesen alten und neuen Autoritätsgläubigkeiten gegenüber tat Haller eines: $\mathrm{Er}$ ließ die Natur selbst sprechen in über 200 Tierexperimenten, von denen zwanzig eigene waren, die übrigen von Schülern und anderen Forschern ${ }^{52}$. Nie vor- und nachher waren in London und Kopenhagen, in Königsberg und Berlin, in Paris, Lyon, Montpellier, in Bologna, Florenz, Rom und Neapel so viele Tiersehnen mit Skalpellen gestochen, durchbohrt, halb und ganz durchtrennt, mit Gewalt herausgerissen, mit Eisen gereizt, mit Höllenstein und Schwefelsäure geätzt und gebrannt worden wie in den Jahren nach 1752. In überwältigender

${ }^{48}$ Die ältere chirurgische Literatur, zusammengestellt in der Dissertation des HallerSchülers Peter Castell Experimenta, quibus varias humani corporis partes sentiendi facultate carere constitit, Gotting. 1753, abgedruckt in A. HALLER, Disputationes chirurgicae selectae, t. V, Lausannae 1756 (zitiert «Disp. chir.»), S. 256-340, und in Op. racc. 1, 125-65. Die betreffenden Angaben in Disp. chir. V, 315 f.; Op.racc. 1, 140 f.

49 Difficult. S. 3 ff.

50 In der in Anmerkung 8 zitierten Ausgabe S. 19.

51 G. v. Swieten, vgl. Anmerkung 42, § 164, S. 240.

52 Vgl. Vorrede zu Elementa, Band 8, ferner Elementa 4, 434 ff.; Op.min. 1, 497 f.; Mém. $4,153 \mathrm{f}$. 
Weise - den 200 Versuchen Hallers und seiner Anhänger stand eine weit geringere Zahl derjenigen der Gegner gegenüber - schien bestätigt zu sein, was Haller selbst schon seit 1746 wußte: Keine Konvulsionen, keine Muskelzuckungen traten auf, ja das Tier zog nicht einmal seine Pfote zurück, gab keinen Laut der Schmerzäußerung von sich. Was man bisher als Empfindung der Sehne angesprochen hatte, mußte offenbar von den neben der Sehne verlaufenden Nerven oder von der Sehnenscheide herrühren ${ }^{52 a}$. Also mußte auch die Achillessehne des Menschen ganz und gar unempfindlich sein.

Des Menschen? fragt de Haen aus Wien zurück ${ }^{53}$. Der clarissimus vir habe seine Experimente doch nur an Tieren angestellt, die sich untereinander essentialiter unterschieden und schon gar erst vom Menschen! «Wie unklug also von Experimenten, die man an Tieren anstellte, auf den Menschen zu schließen! Wie gefährlich ein solcher Schluß, wenn er noch dazu darauf aus ist, die Gesetze des praktischen Handelns zu verändern!»

Nicht nur de Haen hat derartig die Beweiskraft des Tierexperiments für den Menschen geleugnet, auch Giambattista Bianchi (1681-1761) in Turin ${ }^{54}$, so daß Haller ${ }^{55}$ und sein Anhänger in Bologna, MARc' Antonio Caldani (1725-1813) ${ }^{56}$, es für notwendig erachteten, auf die Homologie von Funktion und Bau der Organe bei Tier und Mensch hinzuweisen. Alles dies ist sehr aufschlußreich für die richtige Wertung der Widerstände, auf denen das systematisch von Haller angewandte Tierexperiment in seiner Anfangszeit stieß. Erst wenn man sie berücksichtigt und sich aus den Streitschriften das geistige Milieu dieser frühen Experimentalzeit erschließt, erhalten nicht nur die bisher vernachlässigten Dokumente dieses Streites in der Medizingeschichte den ihnen zukommenden Platz, sondern die Tat Hallers erst ihr historisches Relief. Was im Munde des (hier ${ }^{57}$ ) experimentierfeindlichen de Haens Spott gegen Haller sein sollte, ist für ihn zum un-

${ }^{52 a}$ Vgl. R. СснӓR, Albrecht von Hallers neue anatomisch-physiologische Befunde und ihre heutige Gültigkeit, Berner Beiträge zur Geschichte der Medizin und Naturwissenschaften, Nr.16, Bern 1958, S.44.

53 Difficult. S. $19 \mathrm{f}$.

54 Op. racc. 2, 19.

55 Op. min. 1, 480.

56 Mém. 3, 483.

57 DE HAEN widerspricht sich hier selbst. Er hat bekanntlich selbst zahlreiche Tierexperimente zur Klärung des Ertrinkungstodes angestellt. Vgl. Rat. med. cont. 1, 2, 316 ff.; ferner M. Neuburger, Anton de Haen als Experimentalforscher, Viennensis medica IV, Wien 1898. 
bedingten Ruhm geworden ${ }^{58}$ : «Et hiscene experimentis inaedificata est, quam aeternam volueris, Physiologia!»

Indes ist die Argumentation de Haens und Bianchis gegen die Beweiskraft der Tierversuche Hallers für den Menschen doch vereinzelt geblieben und wurde in der Folge durch 32 an der Achillessehne des Menschen selbst gemachte chirurgische Beobachtungen ${ }^{59}$ außer Kraft gesetzt. In solcher Weise bestätigt, hat Haller es vermocht, daß fortan keine Behauptung mehr aufgestellt und viel weniger noch anerkannt werden konnte, die nicht experimentell begründet war. Indem Haller seine Gegner zwang, die gleichen Waffen zu gebrauchen wie er, eroberte und sicherte er dem Tierexperiment seinen überragenden Platz in den biologischen Wissenschaften.

In diesem Sinne erwiderte Haller auch de Haen in seiner Apologia (S.10), daß dieser niemals selbst gesehen habe, was er behaupte, «sondern die Autorität anderer hast du vorgeführt und (bloße) Meinungen gegen Experimente...» de Haen hatte sich nämlich in seiner Widerlegung ${ }^{60}$ auf mehrere von Ambroise Paré (1510-1590) ${ }^{61}$ beobachtete Fälle gestützt, die bei partieller Achillessehnenruptur eindeutig großen Schmerz und teilweise sogar dauernden Funktionsausfall bezeugten. Er hatte auch einen von Jean Louis Petit (1674-1750), dem führenden französischen Chirurgen der ersten Jahrhunderthälfte, in den Mémoires de l'académie des sciences des Jahres 1722 publizierten Fall von totaler subkutaner Ruptur beider Achillessehnen herangezogen und sich darauf berufen, daß sich hier die proximalen Sehnenstümpfe als ausgesprochen schmerzempfindlich erwiesen hätten. Es ist dies der so berühmt gewordene und in der Literatur des 18. Jahrhunderts viel zitierte Fall des Berufsspringers Cochoix, dessen völlige Heilung auf konservativem Wege (Bandagenbehandlung) Jean Louis Petit geglückt war ${ }^{62}$.

De Haen konnte aber auch Tierexperimente gegen Haller ins Treffen führen, allerdings auch hier nicht eigene, aber immerhin doch solche, die aus der Schule van Swietens hervorgegangen waren, nämlich aus ihrem

\footnotetext{
58 Vindic. 166.

59 Von Haller zusammengestellt in Op. min. 1,496 f.

60 Difficult. $14 \mathrm{ff}$.

61 J.F.Malgaigne, Euvres complètes d'Ambroise Paré, t. II, Paris 1840, S. 110 f. Dazu vgl. E. Gurlt, Geschichte der Chirurgie, 3. Band, Berlin 1898, S. 617.

62 Jean Louis Petit, Traité des maladies des os. Nouvelle éd. par A. Lours, 2. Band, Paris 1772, S. 289-352. Vgl. auch die diesbezüglichen Bemerkungen LouIs' im Discours préliminaire des ersten Bandes.
} 
Prager Zweig. Haller hat auf sie im 4. Band der Elementa wiederholt Bezug genommen und sich in der Réponse générale der Mémoires ebenso wie in deren erweiterter Form, der responsio generalis der Opera minora, mit ihnen, teilweise sogar im einzelnen, auseinandergesetzt. Desohngeachtet haben sie bisher keine Beachtung gefunden. Sie sind aber gleichwohl dieser wert, nicht nur wegen der ursprünglichen Frische ihrer Naturbeobachtung und der Ehrlichkeit ihres noch vielfach unbeholfenen Wollens, sondern auch als ein Dokument des Elans, den van Swieten nicht nur in Wien und auf dem klinischen Sektor, sondern auch in Prag auf dem physiologisch-experimentellen zu entfalten wußte.

Dort hatte er tüchtige Helfer gefunden in dem Iren William Mac Neven (1714-1787), den er zum Fakultätsdirektor machte, und in JoHANN BAPTIST BoHAdsch. Von ihnen bekam unter Zustimmung van Swietens der medicinae candidatus und spätere ärztliche Betreuer böhmischer Militärspitäler, IGnaz Radniczky ${ }^{63}$, als Doktoraufgabe zuerteilt, die Göttinger Versuche zu wiederholen. Was dann 1756 der Prager Fakultät als «Specimen inaugurale medicum» vorgelegt wurde, bestätigte in seinem Untertitel das, woran man in Wien von allem Anfang an nicht gezweifelt hatte: «sistens experimenta quaedam, quibus constitit, eas partes esse sensu praeditas, quibus Hallerus cum aliis quibusdam omnem sentiendi facultatem cum irritabilitate denegat. »

In diesen Versuchen begnügte sich Radniczky nicht damit, die Sensibilität der Sehnen von Hunden sowie sogar eines Pferdes in zehn Experimenten festzustellen und zwei entsprechende Beobachtungen am Menschen beizufügen; er machte sich auch Gedanken darüber, warum die Göttinger Forscher, insbesonders der Haller-Schüler Peter Castell, in seinem 1753 erschienenen «Specimen inaugurale medicum ${ }^{64} \mathrm{zu}$ einem dem Prager so entgegengesetzten Versuchsergebnis gekommen war. So erhalten wir im «Discursus ad Sectionem I» sehr treffende Bemerkungen zur Psychologie des Schmerzes bei Tieren mitgeteilt, derart, daß die gequälten Versuchstiere gelernt hätten, einen dem vorangegangenen (bei Durchtrennung der Haut) neu hinzugefügten Schmerz (bei Inzision der Sehne) nicht durch neue Abwehrbewegungen zu beantworten und ihn dadurch nur zu vermehren, sondern ihn vielmehr durch eine scheinbar reaktionslose Ruhestellung

${ }^{63}$ In dem Brief vom 15. Februar 1772 an Haller (Epist. VI., Nr. 305, S. 86) bezeichnet sich RADNiczKy als Direktor der Militärspitäler in Prag.

${ }^{64} \mathrm{Vgl}$. Anmerkung 48. 
zu vermindern. Abwehrbewegung und Klagegeheul seien daher nicht immer als ein sicherer Indikator der Sensibilität zu werten. Auch könne das Tier in der Vehemenz seines Schmerzes oft einen neuen gar nicht mehr aufnehmen. Ferner gäbe es beim Tier auch eine Art Schmerzgewöhnung. Während nämlich die an den Schnitt des scharfen Skalpells gewöhnten Versuchstiere auf einen neuen nicht mehr reagierten, hätten sie es aber wohl bei Ätzung der Sehnen mit Schwefelsäure oder Höllenstein getan. Wenn man alle diese Momente bei der Auswertung der experimentellen Beobachtungen in Betracht ziehe, sei wohl das entgegengesetzte Ergebnis der Göttinger Versuche erklärlich. Konvulsionen allerdings habe auch er nicht gesehen.

Durch diese Prager Dissertation erhalten wir unmittelbaren Einblick in die Fülle von neuen und noch nicht bewältigten Aufgaben, vor die das von Haller systematisch angewandte Tierexperiment die Forscher nicht nur in Prag, sondern allerorten stellte. Denn dieselben oder ähnliche Fragen hatten auch Robert Whytt (1714-1766) in Edinburgh ${ }^{65}$ und Karl Christ. Krause (1716-1793) ${ }^{66}$ in Leipzig aufgeworfen, und Thomasio Laghi in Bologna fügte die bedeutungsvolle hinzu ${ }^{67}$, ob wirklich mit der Feststellung des Schmerzes der ganze Bereich der Sensibilität erschöpft sei oder ob nicht vielmehr auch die Berührungsempfindlichkeit (tactus) dazugehöre. Man sieht: Alles ist durch die Experimente Hallers in Fluß gekommen. Im lebhaften Hin und Wider der Meinungen werden die Grundlagen der modernen Experimentierkunst erobert, ihre Gesetzlichkeiten festgelegt. Auch hier war es Haller selbst, der die wichtigsten Regeln zusammenfaßte und auf die Fehlerquellen wiederholt ${ }^{68}$ und besonders in der responsio generalis ${ }^{69}$ aufmerksam machte.

Auch Radniczky muß es sich vom Meister des Experiments gefallen lassen, daß er trotz den als notwendig erkannten psychologischen Kautelen eines grundsätzlichen Fehlers überführt wird, der mangelhaften Isolierung der Versuchsbedingungen. So tadelt Haller ${ }^{70}$ an seinem Experiment 5 und 7 der Sectio I, daß er als Sensibilität der Sehnen angesprochen, was in

65 Physiological Essays, Edinburgh 1752.

${ }^{66}$ Prüfung der Preisschrift des Herrn Le Cat von der Muskelbewegung, Leipzig 1755. Dazu vgl. K. Sprengel, a. a. O., S. $404 \mathrm{f}$.

67 Op. racc. 2,328.

68 Elementa 4,440.

${ }^{69}$ Op. min. $1,443.460 .474$.

${ }^{70}$ Op. min. 1, 457; Mém. 4,54. 
Wahrheit nur die Sensibilität der Haut bzw. des benachbarten Nerven bezeuge. Und in ähnlicher Weise zeiht Haller ${ }^{71}$ aueh de Haen der "vaga experimenta». Hier aber erweist seine Kritik eher die Mängel und Grenzen seiner eigenen Sensibilitätsauffassung, die die Innervation bestimmter Gewebe (Sehne, Peritonaeum, Pleura, Periost, Dura mater usw.) nicht erkannte und daher ihre Empfindungsfähigkeit leugnete, als den absoluten Irrtum de Haens. Denn es wird offenbar, daß der Wiener Kliniker sich bereits in einer für seine Zeit außerordentlich fortschrittlichen Weise um die anatomische Lokalisierung des klinisch festgestellten Schmerzes bemühte. Systematisch sezierte er seine Krankenhausfälle, und das nicht erst seit dem Jahr, in dem zugleich mit Morgagnis fachbegründendem Werk seine Difficultates erschienen (1761), sondern, wie wir aus den Opuscula inedita ${ }^{72}$ wissen, bereits Jahrzehnte vorher, wann immer es die Umstände erlaubten. Dieses Bedürfnis nach Verifizierung des klinischen Befundes am Seziertisch ist für die ältere Wiener Schule nicht minder signifikant ${ }^{72 a}$ als das minutiöse Gefühl ihrer klinischen Observanz am Krankenbett. Dieses allein pflegt man zu preisen, jenes zu übersehen. Beide aber gehören in dieser Schule zusammen und verbinden sich in ihr mit einem lebhaften Streben nach rationeller Therapie zu jenem organischen Ganzen, in dem wir den hohen Ruhm dieser Schule als zu Recht begründet erkennen.

Ein Teil dieses Ganzen, de Haens pathologisch-anatomische Bemühungen, wird auch in dem Referat transparent, das Haller ${ }^{73}$ über das Raisonnement des Wiener Klinikers gibt. Gleichzeitig aber präludiert dieses Referat dem Akzent, auf den es in der Auseinandersetzung zwischen Kliniker und Theoretiker ankommt, und zwar in folgender Weise: «Er (sc. de Haen) sagte: Schmerz bestand im Abdomen. Ich (sc. de Haen) habe keine Ursache dafür in der Leiche gefunden, außer einem Fleck im Peritonaeum. Ergo ist das Peritonaeum schmerzempfindlich. Starke Kopfschmerzen bestanden. Ich sezierte die Leiche und fand nichts außer ein Aneurysma der Arterien der Dura mater. Ergo ist diese Membran der Empfindung fähig. Bei der Karies und der Spina ventosa bestehen Schmerzen. Ergo ist das Periost schmerzempfindlich. Es schmerzt bei der Paronychie der Finger. Ergo sind die Sehnen schmerzempfindlich. Der Nierenstein macht Schmerzen. Ergo

71 Apologia $7 \mathrm{f}$.

72 Zitiert in Anmerkung 25.

72a Dazu vgl. den demnächst in der Dtsch. Med. Wschr. erscheinenden Aufsatz Leopold Auenbrugger - Schüler van Swietens.

73 Op. min. 1,460 f. 
sind auch die Nieren einer scharfen Empfindung fähig. Schmerzen bestanden im Abdomen, weil die Milz außerordentlich geschwollen war. Ergo ist die Milz schmerzempfindlich.» Und nun geht Haller in seiner Kritik auf den nervus rerum los, der es hier auch im wahren Sinne des Wortes ist: «Niemals hat der clarissimus vir bedacht, es könne in ein und demselben Teil sowohl der empfindende Nerv als auch die unempfindliche Sehne und die unempfindliche Arterie vereinigt werden ».

Während also für das synthetisierend-organbezogene Denken des Klinikers Periost, Sehne, Milz usw. als eine organische Ganzheit empfindlich sind, sind sie es für das analysierende Denken des Theoretikers als spezifisch fibröses bzw. spezifisch parenchymatöses Gewebe an sich nicht, sondern nur dann, wenn diese Gewebe mit dem «organe unique du sentiment», dem Nerv, nachweislich ausgestattet sind. "Comme la sensibilité croit avec les nerfs, elle s'évanouit avec leur absence, et aucune partie n'est sensible, lorsque l'organe unique du sentiment ne s'y trouve pas ${ }^{74}$. Der grundsätzliche Fortschritt dieses Denkens ist offenbar, aber ebenso, daß er sich in einer Zeit, in der die mikroskopische Technik der Gewebsanalyse noch ganz unentwickelt war, als Rückschritt erweisen mußte. Es war Haller und seinen Anhängern trotz Einsatz des eben von Lieberkühn erfundenen Sonnenmikroskops nicht möglich, im Gewebe der Sehne, der Dura mater usw. nervöse Verzweigungen zu entdecken.

Das physiologisch-experimentell angepackte Problem der Sensibilität war zu einem anatomisch-morphologischen geworden und erweist auch in den hier ins Auge gefaßten Details die Physiologie Hallers als das, was sie eigentlich war, als eine Anatomia animata. Denn die ganze Frage nach Sensibilität und Insensibilität nicht nur der Achillessehne und der Dura mater, sondern aller von Haller untersuchten Membranen und Organe mündet letztlich in die Frage: wie weit kann makro- und mikroskopisch eine nervöse Versorgung der Gewebe nachgewiesen werden?

Hier hakt de Haen ein und kann sich in den Difficultates (S. $15 \mathrm{ff}$ ) auf die Anatomenzelebrität der ersten Jahrhunderthälfte, auf den Lehrer Hallers selbst, auf Jacques Benigne Winslow (1669-1760) in Paris, als Zeugen für die Innervation der Achillessehne berufen. In seiner Exposition anatomique de la structure du corps humain ${ }^{75}$ beschreibt dieser die Verzweigung des N.ischiadicus in der Kniekehle und daß dort ein Ast, Scia-

74 Mém. 4, 28.

75 Paris 1732, Nr. 326-331, S.459. 
tique crurale interne genannt, zwischen dem M.soleus und den großen Zehenbeugern bis an den Malleolus internus verlaufe und auf diesem Verlaufe auch Fasern zur Achillessehne abgebe.

Als de Haen 1761 diese Beschreibung Winslows gegen Haller ins Treffen führte, wußte er noch nicht, daß um sie unter den italienischen Anatomen, Physiologen und Chirurgen eine heftige Debatte bereits im Gange war und schon eine Fülle von neuem Material vorlag. Thomasio Laghi in Bologna hatte 1756 bei Tieren und zusammen mit dem Anatomen Borghi auch beim Menschen die Verzweigung des «nervus cruralis posticus» (= N.tibialis) eingehend studiert und war zu dem auch zeichnerisch festgehaltenen Ergebnis gelangt, daß der Nerv in der Nähe des Fersenbeins auch einige Äste zur Achillessehne abgebe ${ }^{76}$. Diese Beobachtungen wurden im selben Jahre auch von Domenico VAndelli (1735-1816) ${ }^{77}$ in Padua genauer dahin bestätigt, daß der Nerv sowohl zur Scheide der Achillessehne als auch in ihre eigentliche Substanz Zweige entsende. Das widersprach aber genau dem, was Urbano Tosetti ${ }^{78}$ aus Rom an Haller über das Untersuchungsergebnis des dortigen Primarchirurgen am Spital San Giovanni in Laterano, Luigr Paliani, mitteilen konnte: Kein Nervenast trete in die Substanz der Sehne wirklich ein, teils endigten sie in den benachbarten Teilen, teils setzten sie sich gegen den Fuß zu fort. Dasselbe bestätigten die Bologneser Gelehrten und Anhänger Hallers, Marc' Antonio Caldani und Felice Fontana (1728-1805), $1757^{79}$.

Was half es da, daß de Haen sich in seiner zweiten Streitschrift, in den Vindiciae (S. 133 f.), 1762 noch einmal auf Winslow und auch auf Vandelli berief? Die neuen Autoritäten in Göttingen und Bologna hatten gesprochen und die Achillessehne war auch anatomisch insensibel erklärt.

Dasselbe Schicksal wurde auch der Dura mater zuteil, deren Sensibilität ebenfalls im Brennpunkt des Streites stand. Auch hier hatte Thomasio Laghi ${ }^{80}$ die Angaben bei Lorenz Heister und J.B. Winslow bestätigt gefunden, daß nämlich der 5. und 7. Hirnnerv Äste zur harten Hirnhaut entsendeten. Auch Giambatrista Morgagni (1682-1771) und sein Schüler Domenico Cotugno (1736-1822) traten für die sensible Versorgung der Dura mater ein. Ihre Auffassung konnte sich jedoch gegenüber derjenigen der Haller-

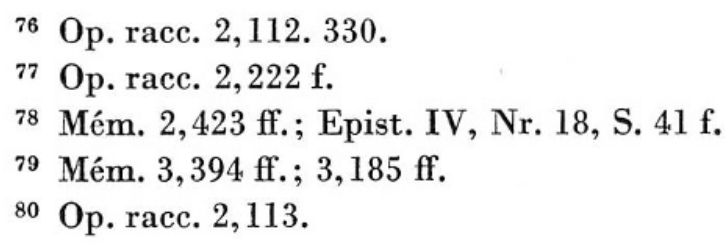


schüler Johann Friedrich Megkel (1724-1774) ${ }^{81}$ und Johann GottFRIED ZINN (1727-1759) ${ }^{82}$, nicht durchsetzen, die, wie auch Haller ${ }^{83}$ selbst, keine Nerven in der Dura mater nachweisen konnten. Auch Caldani und Tosetti ${ }^{84}$ hielten die Dura mater für nervenlos, und als auch Samuel Thomas von Sömmering (1755-1830) sich in seiner grundlegenden Arbeit De basi encephali et originibus nervorum cranio egredientium ${ }^{85} 1778 \mathrm{zu}$ dieser Meinung bekannte, da blieb sie bis über das 18. Jahrhundert hinaus die herrschende.

Eine positivistische oder pragmatische Geschichtsbetrachtung könnte an diese Beispiele aus der Sensibilitätslehre Hallers die Sentenz anfügen: Wie oft doch Irrtümer eines großen Mannes eine lange Zeit hindurch den Fortschritt der Wissenschaften hemmen! Man kann aber den vorgelegten historischen Befund auch anders werten, nicht nur prospektiv, auch retrospektiv, indem man ihn beispielsweise unter dem Aspekt des Hegelschen Gesetzes von Thesis, Antithesis und Synthesis betrachtet. Wenn man dies tut - und es erscheint mir zur Erhellung des problemgeschichtlichen Ablaufes nützlicher zu sein -, dann erscheint die Negierung der Sensibilität der Dura mater durch Haller als die notwendige Antithesis auf die vorausgegangenen Thesen Antonio Pacchionis (1665-1726) und Giorgio Baglivis (1669-1707), die in der harten Hirnhaut schlechtweg das empfindende und bewegende Zentralorgan des Körpers erblickten ${ }^{86}$; und im gleichen Sinne stellt sich die Negierung der Sensibilität der Sehnen, Aponeurosen und Bänder als notwendige Antithesis dar, um die Thesis der Antike, Nerv = Sehne = Band, endgültig und nicht nur im Morphologischen, sondern auch im Funktionellen zu überwinden. Das ist also durch

81 Ioann. Fred. Meckel, Tractatus de quinto pare nervorum cerebri, Gotting. 1748, S.18, $21,54 \mathrm{f}$.

82 IoANN. GotTr. ZinN, Diss. inaug. med. sistens experimenta quaedam circa corpus callosum, cerebellum, duram meningem in vivis animalibus instituta. Abgedruckt in: Alb. HALler, Disputationes anatom. select., vol. VII, Gotting. 1752, S. $459 \mathrm{ff}$.

83 Elementa 4,138 f.; Op. min. 1,445.

84 Mém. 4,3,433 ff.; $2,477 \mathrm{f}$.

${ }^{85}$ SАм. Тном. Sömmering, De basi encephali et originibus nervorum cranio egredientium, Gotting. 1778.

${ }^{86}$ Dazu vgl. M. Neuburger, Die historische Entwicklung der experimentellen Gehirn- und Rückenmarksphysiologie vor Flourens, Stuttgart 1897, S. 76 ff. Е. BASTHOLM, The History of Muscle Physiology, Acta Hist. Scient. nat. et med., Vol. VII, København 1950, S. $178 \mathrm{ff}$., E. Lesky, Giorgio Baglivi und die Konstitutionslehre, Sudh. Arch. Gesch. Med. Naturwiss. 35 (1953) $289 \mathrm{ff}$. 
Haller geschehen. Und darin möchten wir die Bedeutung seiner Sensibilitätslehre erkennen und hoffen, daß es ihr künftig in der Medizingeschichte nicht nur beschieden sei, gerade noch als ein Adnex der die Zukunft der Muskellehre tragenden Irritabilitätstheorie geduldet zu sein.

Die Synthesis, um das Bild vom Dreitakt der Hegelschen Geschichtsphilosophie wenigstens für das Problem der Innervierung der Achillessehne durchzuführen, brachte eine viel spätere Zeit. Denn nachdem die Epoche Hallers ihre Mittel an ihr erschöpft hatte, ruhte diese Frage lange, wenn wir von den knappen Angaben in Albert Koellikers (1817-1905) Handbuch der Gewebelehre des Menschen ${ }^{87}$ absehen, daß «die Achillessehne und Sehne des Quadriceps nur Gefäßnerven » besäßen. Es ist kein Zufall, daß fast gleichzeitig 1875 die Frage von $\mathrm{H}$. SACHS ${ }^{88}$, 1876 von dem Brücke-Schüler und Grazer Physiologen Alexander Rollett (1834-1903) ${ }^{89}$ und 1878 von CAmillo Golgi ${ }^{90}$ zentral wieder aufgenommen wurde. Denn die Entdeckung der Sehnenreflexe 1872 durch Wilhelm Erb und Carl Westphal hatte sie von neurologischer Seite aus in den Mittelpunkt des Interesses gerückt. An der Sehne des M.sternoradialis des Frosches fand denn auch Rollett mit einer fortgeschritteneren Gewebstechnik die Sehnenspindeln und in ihren Verästelungen die nervösen Endapparate ${ }^{91}$. Aber auch ihm war es weder chemisch noch mechanisch möglich, von der Sehne aus Muskelkontraktionen auszulösen. Damit ist in diesem Punkt das negative Reizungsergebnis bestätigt, das die Physiologen des 18. Jahrhunderts zu der Lehre von der Insensibilität der Sehnen verleitet hatte. Die endgültige Klärung der Achillessehnen-Innervation beim Menschen brachte 1927 die Arbeit von J. J. TschuRAJEW ${ }^{92}$. In dieser konnte er in $91 \%$ der Fälle mit der modifizierten Schultzeschen Methode die von den Rr. calcanei med. des N. tibialis gebildeten Endapparate nachweisen und gleichzeitig das Versuchsergebnis Rolletts bekräftigen, daß diese Endigungen in keiner Beziehung zu den

${ }^{87}$ Leipzig 1852, S. 185.

${ }^{88}$ H. SACHS, Die Nerven der Sehnen, Arch. Anat., Physiol. wiss. Med. 1875, 402-16.

89 A. Rollett, Über einen Nervenplexus und Nervenendigungen in einer Sehne, Sitzber. k. Akad. Wiss. Wien. Math.-nat. Kl. 73 (1876), 3. Abt., S. 34-53.

90 C. GoLGI, Intorno alla distribuzione e terminazione dei nervi nei tendine dell'uomo e di altri vertebrati, R. Istit. Lomb. d. scienz. e lett., Rendiconti, Milano 1878, 2. s., XI, S. 445-53.

${ }^{91}$ Vgl. auch J. Schaffer, Lehrbuch der Histologie und Histogenese, Wien 1933, S. 255.

92 J. J. TschuraJew, Die Innervation der großen Sehnen der unteren Extremität des Menschen (Die Sehne des M.quadriceps, Lig. pat., Retinac. patellae, Tendo Achillis) nebst ergänzenden Befunden zur Anatomie des N.femoralis, Gegenbaurs Morph. Jb.58, (1927) 1-42. 
Sehnenreflexen stehen. Damit ist die Synthesis unseres Problems vollzogen und stellt sich einerseits in der Bestätigung des negativen Ergebnisses der Reizungsversuche des 18. Jahrhunderts dar, anderseits aber auch in deren Korrektur. Denn ungeachtet dieses negativen Ergebnisses wurde gleichwohl das Vorhandensein nervöser Endapparate in der Achillessehne bewiesen und als deren Funktion «die Apperzeption der allgemeinen Sensibilitätsreize» sichergestellt ${ }^{93}$.

Für uns ist damit das Problem der Hallerschen Insensibilität der Achillessehne in befriedigender Weise zum Austrag gekommen. Aber wie konnte sich der Praktiker und Theoretiker des 18. Jahrhunderts darüber und über die anderen als insensibel erklärten Gewebe verständigen? Eine Verständigung, ja eine Aussöhnung zwischen Haller und de Haen kam nämlich durch die Vermittlung des beiderseitigen Freundes und Genfer Philosophen Charles Bonnet (1720-1793) ${ }^{94}$ zustande und war auch möglich geworden durch die Interpretationen, die Haller 1766 in der Vorrede des 8. Bandes der Elementa zu seiner Lehre gab. Dort eröffnet er folgende Möglichkeiten: «Wenn man an den Sehnen jemals Nerven zeigen wird, so glaube ich, daß sie nur ein stumpfes Gefühl haben, als sich für die wenigen Nerven schickt; so kriechen auf den Gelenkkapseln und auf den Knochenhäutchen Nerven, welche ihre Empfindlichkeit mit diesen Bekleidungen theilen können. Streuen sie in diese Theile einige Äste aus, so wird auch, nach Proportion dieser Nerven, an den Kapseln und am Knochenhäutchen eine dunkle Empfindung vorkommen. Sie wird sowohl an diesen Bekleidungen als an den Sehnen bei einer Entzündung größer werden, so wie dieses Übel allenthalben am Körper die Empfindung auf einen höheren Grad treibt ... daß ferner das Ribbenfell zwar ohne Empfindung seyn, die auf dessen convexen Rücken aber liegenden Nerven, wofern sie etwa von einer Entzündung einer benachbarten Membran ergriffen werden, heftig schmerzen, alles dieses stimmet sowohl mit der Speculation der Vernunft, als mit den Versuchen überein.»

Mit diesen Auslegungen konnte sich auch der Kliniker zufrieden geben, und er hat es auch getan, indem de Haen nicht nur brieflich ${ }^{95}$, sondern auch öffentlich in seinem Hauptwerk, der Ratio medendi ${ }^{96}, 1768$ erklärte: «Was

93 J. J. Tschurajew, a.a. O., 38.

94 Dazu vgl. den bei J.StroHL, Albrecht von Haller, Gedenkschrift 1938, S. 19, abgedruckten Brief Hallers, sowie Rat. med. 12,7,393.

${ }_{95}$ Epist. VI, Nr. 280, S. 15 ff.

96 Rat. med. 12, 7,394, f. 
kann wohl wahrer und deutlicher seyn als dieses, und wie kann man sich offenherziger erklären?... Denn was hindert es in der Praxis, ob das Rippenfell, die dicke Hirnhaut, die Häute, die Bänder, die Gelenkkapseln und die Sehnen ihrer eigenen Nerven wegen Empfindung haben oder ob Nerven anderer Theile, die über oder unter oder durch besagte Theile gehen, die Ursache ihrer Empfindlichkeit sind: wenn selbige nur bey Verwundungen, Quetschungen und sonst ... eben die Hülfsmittel erfordern, welche die nervigen Theile mehrentheils verursachen und erfordern.»

Diese Aussöhnung, von der Haller sich zunächst nicht sicher war ${ }^{97}$, ob sie de Haen auch ehrlich meinte, hat in der Gelehrtenwelt großes Aufsehen erregt. Wir wissen aus einem Brief Caldanis ${ }^{98}$, daß die Anhänger Hallers befürchteten, er würde seine Lehre abschwächen. Indes ist dies nicht mehr geschehen, als aus der eben ausgeschriebenen Stelle hervorgeht. Wir haben auch keinen Grund, zu zweifeln, daß diese Aussöhnung eine echte war, und Haller hat sie auch in der Folge als eine solche genommen. Häufig gehen nach 1768 Briefe zwischen Wien und Bern hin und her ${ }^{99}$, in denen nicht nur Gelehrtes, sondern auch Persönliches zur Sprache kommt. Ja, als es im Herbst 1773 mit Hallers Gesundheit nicht zum Besten steht ${ }^{100}$, läßt er sich sogar von de Haen beraten, und dieser tut es ausführlichst und bereitwilligst ${ }^{101}$ trotz der Arbeitslast seines Tages, die den nun schon müde und selbst krank Gewordenen zu überwältigen droht. Als es schließlich im Frühjahr 1776 mit ihm wirklich zu Ende geht, da schreibt Haller in einem Brief an Gesner ${ }^{102}$ die Schlußworte zu diesem Kapitel seines Lebens. Es ist nur eine kurze Äußerung, aber diese kommt ganz aus der Mitte des Menschen, eines Menschen, der immer ein grand homme geblieben ist, auch seinem Gegner gegenüber, und als solcher den Frieden mit ihm zu den «feliciora eventa» seines eigenen Lebens zählt.

97 H. E. Sigerist, Albrecht von Hallers Briefe an Johannes Gesner (1728-1777), Abh. d.

k. Ges.d. Wiss. Götting. Math.-phys. Kl., N. F. Band XI/2, Berlin 1923, S. 410, Brief Nr. 365 vom 16. April 1768, und S. 426, Brief Nr. 390 vom 3. Mai 1769.

${ }_{98}$ Epist. VI, Nr. 282, S. 20 f.

99 Veröffentlicht sind nur die Briefe de Haens an Haller. Vgl. Epist. V, Nr. 272, S. 331 f.;

Nr. 275, S. 338 f.; VI, Nr. 280, S. 15 f.; Nr. 325, S. 126 ff., Nr. 330, S. 148 ff.

100 H. E. Sigerist, a. a. O., S. 488. Brief Nr. 481 vom 25. September 1773.

101 Epist. VI, Brief vom 13. Oktober 1773, Nr. 326, S. $129 \mathrm{ff}$.

102 H. E. Sigerist, a. a. O., S. 518, Brief vom 17. Februar 1776, Nr. 519. 


\section{Chirurgische Folgerungen}

Anton de Haen hat schon recht gesehen ${ }^{103}$, daß durch die Versuchsergebnisse Hallers «die chirurgische Praxis in Behandlung der Wunden und Geschwüre ... ganz anders als bisher getrieben» wurde. Von Hallers Sensibilitäts- oder besser Insensibilitätslehre ist in der Tat ein mächtiger Impuls auf die Chirurgie seiner Zeit ausgegangen. Ihn nach all seinen Aspekten einigermaßen erschöpfend darzustellen, würde weit das Ziel überschreiten, das sich diese Untersuchung gestellt hat. Und doch möchte sie nicht darauf verzichten, in einem bisher so wenig bearbeiteten Feld Hallerscher Ausstrahlung wenigstens an einem Beispiel, dem gewählten der Achillessehne bzw. der Sehnenwunden, die Bedeutung Hallers für die zeitgenössische Chirurgie zu exemplifizieren.

Freilich hat es schon vor Haller so kühne Chirurgen gegeben - Haller führt sie in seinem Akademievortrag 1752 (S. 17) und später wiederholt ${ }^{104}$ als seine Zeugen auf - wie Job van Meekeren, Jan Daniel Schlichting, Bryan Robinson, George Thompson u. a., die bei offenen Wunden Sehnen mit der Zange faßten und sie durchschnitten, ohne dabei jene saevissima mala zu sehen: Fieber, Entzündung, Gangrän, Konvulsionen und Tod. Ja, es hat sogar Wundärzte gegeben - und das schon seit dem 16. Jahrhundert -, die planmäßig mit der Nadel das zerrissene Sehnengewebe durchstachen, um es nämlich zu nähen. In der französischen Ausgabe der Heisterschen Chirurgie $^{105}$ sind eine ganze Reihe von solchen Pionieren der Sehnennaht genannt, der Schweizer Felix Würtz ${ }^{106}$ (um 1500/10-1590/96), JohaNN Vesling (1598-1649) in Padua, Marc' Aurelio Severino (1580-1656) in Neapel, Jean Bienaise (1601-1681) in Paris, Anton Nuck (1650-1692) in Leiden u.a. Sehr richtig bemerkt dazu der Haller-Schüler Peter Castell 1753 in seiner Dissertation ${ }^{107}$, daß alle diese Männer durch dieses ihr Tun «stillschweigend einbekannt hätten, daß die Sehnen ohne Gefahr gestochen und geschnitten werden können. » Das alles hatte man also schon vor Haller an den Sehnen beobachtet und getan und es als die bestaunte Ausnahme auch aufgezeichnet. Und nun war Haller mit seinen Schülern gekommen und hatte die verstreuten Einzelbeobachtungen der niederländischen, eng-

103 Rat. med. 12,7,395.

104 Op. min. 1,449; Elementa 4,428; Op. racc. 1,141 ff.

105 PAUL, Institutions de Chirurgie de M. Laurent Heister, tom. II, Avignon 1770, S. 548.

106 Die einschlägige Stelle abgedruckt bei E. GurLt, a.a.O., 3. Band, S. 252.

107 Vgl. Anmerkung 48. Disp. chir. V, S. 315 f.

108 Vgl. Anmerkung 8, S. 19. 
lischen, französischen, italienischen und deutschen Chirurgen systematisch im Tierversuch überprüft und die bestaunte Ausnahme als die Regel in die Hände der Chirurgen wieder zurückgelegt ${ }^{108}$ : «Wir wollen also unsere Furcht vor den Wunden der Sehnen ablegen, sie mögen gestochen, getrennt, gehauen oder geschnitten sein », war der Satz, der auf diesem Gebiet der Wundchirurgie neue Wege wies.

Als Haller diesen Satz aufstellte und seine Schüler, vor allem Peter Castell und Johann Georg Zimmermann (1728-1795) ${ }^{109}$, ihn durch ihre Experimente im einzelnen untermauerten, da machten sie sich auch Gedanken darüber, welche pathologischen Fakten die bisherige Furcht vor den Sehnenwunden genährt haben könnten. Als erstes führen sie mißdeutete Aderlaßerfahrungen an. Wir können uns ja heute kaum mehr eine Vorstellung machen, in welch ungeheurem Ausmaß die Venaesectio die Therapie der älteren, humoral bestimmten Medizin beherrschte und wie viele Zwischenfälle und selbst schwerste Komplikationen hiebei und zumal durch die Außerachtlassung aller Regeln der Asepsis auftraten. Da konnte es vorkommen, wie in dem durch Ambroise Paré berühmt gewordenen und von Haller ${ }^{110}$ und anderen viel zitierten Fall König Karls IX. von Frankreich, daß ein unvorsichtiger Wundarzt den vor der Medianvene verlaufenden Mediannerv mit seiner Lanzette verletzte. Oder es kam zu einer ausgedehnten Blutung in das umgebende Zellgewebe, zu dessen Entzündung, Vereiterung und Gangrän, mit einem Wort zur Wundinfektion mit all ihren Folgen ${ }^{111}$. Davon konnte ebenso die Hand wie der Fuß und der Unterarm betroffen sein, die Prädilektionsstellen des Aderlasses ${ }^{112}$, an denen überall neben den Gefäßen die großen Sehnen der Beuger verliefen. Und so schrieb man denn alle diese Aderlaßkomplikationen bis zur Sepsis der nach dem alten Dogma, Nerv = Sehne, behaupteten hochgradigen Empfindlichkeit der letzteren zu.

Weiters hat man böse Erfahrungen mit den Panaritia tendinosa, bei denen man Fieber, Schüttelfröste bis zu ausgesprochen septischen Symptomen beobachten konnte, ebenfalls allein auf die hochgradige, dem Nerv gleich geachtete Sensibilität der Sehnen gesetzt. Und die gleiche erkannten

${ }^{109}$ In seiner in Op. racc. 1,74 ff. abgedruckten Dissertatio de irritabilitate, Gotting. 1751, die Stelle Op. racc. 1,83 .

110 Vgl. Anmerkung 8, S. 19; Mém. 4,82.

111 Vgl. die Ausführungen "Von den Zufällen der Aderlaß» bei L. Heister, Chirurgie, Nürnberg 1724, S. 374 ff.

112 Ausführlich besprochen bei G. Heuermanv (vgl. Anmerkung 44) 3, $257 \mathrm{ff}$. 
noch Boerhaave und van Swieten ${ }^{113}$ den Aponeurosen und Faszien der großen Bauchmuskeln zu und machten sie in erster Linie für alle Komplikationen verantwortlich, welche bei Blutansammlungen unter ihnen auftraten. Wahrlich, was sich da durch blinde Autoritätsgläubigkeit im Laufe der Jahrhunderte unter dem Titel «Sensibilitas tendinum» als Einheit zusammengefunden hatte, mußte sich vor den kritischen Blicken Hallers und seiner Schüler als eine ätiologisch sehr divergente Vielheit erweisen. Diese haben also die Göttinger erstmals aufgespaltet, indem sie feststellten ${ }^{114}$, daß einerseits Vereiterung und Gangrän des Gewebes nach Aderlaß oder Bauchdeckenverletzung oder bei Panaritien ursächlich nicht mit der spezifischen Empfindlichkeit des verletzten fibrösen Gewebes, sondern mit dem zwischen diesem Gewebe ergossenen und veränderten Blut zu verbinden sei, anderseits der akute, unmittelbar bei einem Aderlaß auftretende Schmerz wie im Falle Karls IX. nicht von der Sehne, sondern von dem verletzten Nerv stamme. Indem sie diese Unterscheidungen vollzogen, haben sie dem Nerv gelassen, was ihm gehörte, und den Sehnen genommen, was ihnen nicht zukam, nämlich in erster Linie die behauptete hochgradige Schmerzhaftigkeit und primäre Gefährlichkeit ihrer Wunden.

Begierig griffen allerorten die Wundärzte nach dieser neuen Erkenntnis, besonders aber in Frankreich. Der als Übersetzer sehr rührige südfranzösische Chirurg Paul gibt im Anhang zu seiner Ausgabe der Heisterschen Chirurgie $^{115}$ einen Überblick über die größten chirurgischen Errungenschaften des 18.Jahrhunderts. Unter diese Prominenz der Tat stellt er Bordenaves neue von Haller inspirierte Methode der Behandlung der Sehnenwunden bzw. Sehnenerkrankungen ${ }^{116}$. Tatsächlich hat Toussaint Bordenave (1728-1782) in Paris sehr schnell und sehr entschlossen die Konsequenzen aus den Hallerschen Versuchen zu Ende gedacht, indem er folgendes Raisonnement anstellte ${ }^{117}$ : Die bisher behauptete Empfindlichkeit

113 G. v. Swieten (vgl. Anmerkung 42), § 165, S. 242. Dazu vgl. die Kritik J. G. ZimmerMANNS, Op. racc. $1,83 \mathrm{f}$.

114 Disp. chir. V 318; Op. racc. 1,83.

115 Im Anhang zu dem ersten Band des in Anmerkung 105 zitierten Werkes: Mémoires pour servir à l'histoire de la chirurgie du XVIII siècle, et de supplément aux institutions chirurgicales de M. Heister, 1. Teil, Avignon 1773.

116 PAUL, a.a.O., S. X.

117 In einem 1762 in der Académie Royale de Chirurgie gehaltenen Vortrag, den PaUL。 a.a.0., S. 62 f, im Auszug wiedergibt. Vgl. auch Bordenave, Remarques sur l'insensibilité de quelques parties, Paris 1756, und die aus dem Mercure de France (Juin 1757) in Mém. 3,245-68, referierten Experimente. 
der Sehnen und Aponeurosen kann also nicht für die bekannten accidents affreux verantwortlich gemacht werden; diese sind vielmehr die Folge einer Entzündung der fibrösen Gewebe, die ihrerseits durch die Abschnürung der Gefäße (étranglement des vaisseaux) durch sie verursacht werde. Bordenave überträgt hier die zu seiner Zeit geltende pathogenetische Theorie der Gangrän, wie sie FrançoIs Quesnay (1694-1774) ${ }^{118} 1749$ in seinem Traité de la gangrène entwickelt hatte, auf die entsprechenden Verhältnisse bei Sehnenwunden und fordert wie dieser, daß die angenommene Abschnürung der Gefäße durch Schnitte in den entsprechenden fibrösen Gewebsteilen aufgehoben werde, um den bei diesen Wunden beobachteten und gefürchteten Komplikationen vorzubeugen. Bis jetzt hatte man nämlich geglaubt - und das seit der Antike -, dies dadurch tun zu können, daß man siedendes Terpentinöl in die Sehnenwunde goß. Was hat man damit anders bezweckt, so fragt jetzt Bordenave, als die behauptete Sensibilität der Sehnen zu zerstören? Diese besteht aber von vorneherein nicht. Also : «L'instrument tranchant, conduit par les lumières anatomiques, est ici de la plus grande ressource.» Indem Bordenave mit diesem Satz das aktive Vorgehen bei eitrigen Komplikationen der Sehnenwunden und bei Panaritien zur Methode der Wahl erhebt, hat er auch im Praktischen die letzten Konsequenzen aus der Hallerschen Lehre gezogen und durch sie einen weiteren Glaubenssatz der Antike erschüttert, den an die wunderbare Wirkung des siedenden Terpentinöls.

Alle diese Chirurgen und Aktivisten der Hallerschen Sensibilitätslehre, wie Bordenave in Paris und der schon genannte Luigi Paliani in Rom, zu denen auch Georg Heuermann (1722-1768) in Kopenhagen, Ferdinand Wilhelm Mühlmann in Königsberg, Giambattista Verna in Turin, Pier Antonio Perenotti (1732-1797) in Neapel, Pietro Paolo Molinelli (1702-1764) in Bologna, Etienne Jean Pierre Housset (†1810) in Auxerre, JAC. FARJoN in Montpellier u.a. gehörten, sind durch ihre intensive Beschäftigung mit Hallers Lehre vielfach auch selbst namhafte Physiologen geworden. Für sie alle gilt, was nachmals Marie Jean Pierre Flourens (1794-1867) ${ }^{119} \mathrm{im}$ besondern von den französischen Chirurgen der Zeit aussagte: «Man kann die lange Reihe (ihrer) Beobachtungen nicht durchstudieren, ohne zu glauben, man habe es mit physiologischen For-

118 Es wurde die Ausgabe Paris 1771 benutzt. Dort das 9. Kapitel über «l'étranglement », S. $86 \mathrm{ff}$.

119 Zitiert nach M. Neuburger (vgl. Anmerkung 86), S. 194. f. 
schungsergebnissen zu thun, und umgekehrt erscheint der physiologische Versuch zum Theil als chirurgische Operation, aber als eine neue und originelle Operation, zu welcher die praktische Kunst nicht gelangt wäre.» Denn alle diese chirurgischen Avantgardisten physiologischer Lehre haben die Sätze des Göttinger Meisters nicht kritiklos übernommen, sondern sich mit ihnen selbständig in eigenen Versuchsreihen ${ }^{120}$ auseinandergesetzt.

Das hat auch der führende dänische Chirurg der Zeit, Georg Heuermann, in Kopenhagen getan, und zwar so gründlich, daß ein ganzes Handbuch der Physiologie ${ }^{121}$ daraus wurde. In diesem bestätigte er zwar die Versuchsergebnisse Hallers hinsichtlich der Insensibilität der Sehnen, Bänder und Aponeurosen ${ }^{122}$; für die Selbständigkeit seines Beobachtens und Denkens zeugt es aber, da $\beta$ er hinsichtlich der Dura mater zu ganz anderen - und wie wir heute wissen - richtigeren Feststellungen als Haller gelangte, indem er ihm gegenüber die Empfindlichkeit der harten Hirnhaut vertrat ${ }^{123}$. Alle diese Versuchsergebnisse hat Heuermann ganz unmittelbar in seiner dreibändigen Abhandlung der vornehmsten chirurgischen Operationen am menschlichen Körper (Kopenhagen und Leipzig 1754-1757) in die Praxis des Chirurgenalltags hineingenommen und im besonderen für die Behandlung der Sehnenwunden und Panaritien die entsprechenden Folgerungen gezogen, indem auch er wie Bordenave in Paris ein aktives Vorgehen empfiehlt und ihre primäre Ungefährlichkeit vertritt ${ }^{124}$ : «Es sind dahero die Verletzungen der flechsigten und ligamentösen Teile nicht so gefährlich empfindlich und schwer zu heilen, wie man von ihnen geglaubet, sondern können ohne Empfindung verletzet und ebenso leichte wie andere Teile curiret werden, wenn sie zertrennet.»

Es ließe sich nun bis zur Eintönigkeit aus den Schriften der früher genannten Chirurgen erweisen, wie dieser Satz von der primären Ungefährlichkeit der Sehnenwunden Allgemeingut der wissenschaftlich betriebenen Chirurgie der zweiten Hälfte des 18. Jahrhunderts wird. Folgerichtig tritt aber auch noch eine andere Erkenntnis immer klarer hervor, daß nämlich die so lange gefürchteten Zufälle, die accidents affreux, die saevissima mala der Sehnenwunden, zwar noch unbekannter, aber ausschließlich sekundä-

120 Vgl. die im 2. und 3. Band der Mém. referierten Experimente.

121 Kopenhagen und Leipzig 1751-1755.

122 Vgl. auch Mém. 2,111-22.

123 Physiologie 1,131 f.; dazu die Kritik Hallers, Mém. 2,121 f.

${ }^{124}$ Abhandlung der vornehmsten chirurgischen Operationen am menschlichen Körper, 1, S. 49 . 
rer Natur sind. Es ist für den Wandel, den das naturwissenschaftliche Denken nach und durch Haller erfahren hat, bezeichnend, daß diese Erkenntnis von dem führenden deutschen Chirurgen der zweiten Jahrhunderthälfte, von dem Göttinger Professor August Gotтlob Richter (1742-1842) ${ }^{125}$, in seinen Anfangsgründen der Wundarzneykunst mit all der Vorsicht vorgetragen wird, die die inzwischen erlangte Einsicht in die Komplexität vitaler Vorgänge gebot: «Man kann also die nächste Ursache dieser fürchterlichen krampfhaften Zufälle nicht immer und nicht hinreichend bestimmen. Alles, was man davon mit Sicherheit weiß, ist dies: $\mathrm{Da} \beta$ sie sich vorzüglich zu Wunden, gemeiniglich zu Stichwunden flechsichter Theile, am allerhäufigsten zu Verletzungen an den Fingern und Fußzehen ... gesellen. Selten aber ist die Wunde allein Ursache, gemeiniglich bringt sie in Vereinigung mit anderen Ursachen diese Zufälle hervor. Die gewöhnlichsten sind gehinderte Ausdünstung und eine faulichte Beschaffenheit der Säfte.» Hier wird eine Ätiologisierung herangezogen, mit der die zeitgenössische Medizin auch den Hospitalbrand verbindet, und es ist kein Zufall, daß im anschließenden Kapitel Richter zentral zu diesem übergeht.

Mit dieser Zuordnung der saevissima mala der Sehnenwunden ist das jahrhundertealte Dogma von ihrer spezifischen und primären Gefährlichkeit in der wissenschaftlich betriebenen Chirurgie abgewertet. Damit ist zugleich ein Teil Hallerscher Aufklärungsarbeit auf dem Gebiete der Wundchirurgie bezeichnet, aber noch durchaus nicht der Reichtum an Anregungen erschöpft, die von seinen und seiner Schüler Versuche auch auf die konservative Therapie der Sehnenverletzungen ausgingen. Um auch diesen Teil Hallerscher Wirkung ins rechte Licht zu setzen, empfiehlt es sich, wiederum von den Versuchen seines Schülers Castell auszugehen.

Dieser hatte nämlich nicht nur die Sensibilitätsverhältnisse der Sehnen überprüft, sondern auch ihrem Regenerationsvermögen und der Wiederherstellung ihrer Funktion sein Augenmerk zugewendet. Besonders die in den $\S \S$ XVII und XVIII mitgeteilten Versuche ${ }^{126}$, in denen er bei partiell durchtrennten Achillessehnen seiner Versuchstiere eine völlige Wiederherstellung der Funktion beobachten konnte, hatten ihm nach der Obduktion gezeigt, daß es zu einer Wiedervereinigung und Festigung des Sehnengewebes aus eigener Kraft (propria virtute) gekommen war. Ähnliches hatte sich auch Castells Prager Opponenten, Ignaz Radniczky, bei seinen

125 Wir benutzten die 2. Auflage, Göttingen 1787. Dort § 403, S. 244.

126 Disp. chir. V 305. 
Versuchen ergeben ${ }^{127}$, doch hatte er daraus weit weniger radikale Schlußfolgerungen gezogen als Castell. Dieser glaubte nämlich, alles der Heilkraft der Natur überlassen zu können, und konnte sich dabei sogar auf chirurgische Erfahrungen berufen, die der sehr angesehene Bologneser Chirurg, Pietro Paolo Molinelli, bei Fällen von total durchtrennter Achillessehne gemacht hatte ${ }^{128}$. Bei ihnen war es ohne Anwendung von Naht oder Bandagen zur vollen Funktionsherstellung gekommen. Dementsprechend folgerte jetzt Castell: 1. eine Sehnennaht sei bei Verletzungen und Rupturen der Achillessehne überhaupt nicht notwendig und 2. selbst für eine Bandagenbehandlung, wie sie Jean Louis Petit im Falle der totalen subkutanen Ruptur der Achillessehne bei dem Springer Cochoix mit solchem Erfolg angewendet und seither in Form seines Stiefels ${ }^{129}$ propagiert hatte, brauche man weniger Sorge zu tragen.

Gian Vincenzo Petrini ${ }^{130}$, Mathematik- und Philosophieprofessor am Collegio Nazareno in Rom, ist vielleicht doch etwas zu optimistisch gewesen, als er aus diesen Erfahrungen Castells ganz neue Aspekte für eine alteingefahrene Kriegspraktik eröffnete. Bisher hatte man Pferde nur mit durchschnittenen Fesseln dem Feind überlassen und dieser mußte die so unbrauchbar gemachten töten. Nach der neuen Erkenntnis ist das nicht mehr notwendig, verkündet Petrini. Nur ein paar Wochen Ruhe zur Regeneration ihrer Sehnen, und die Rösser werden wieder laufen wie zuvor. Wir wissen nicht, ob die Strategen des 18. Jahrhunderts von dieser Empfehlung Gebrauch gemacht haben.

Man wird auch nicht sagen können, daß sich die Chirurgie der zweiten Jahrhunderthälfte die Folgerungen Castells genau so zu eigen gemacht habe, wie sie ausgesprochen wurden. Auch nicht hinsichtlich der Sehnennaht, die im selben Moment als unnotwendig erklärt und tatsächlich auch verworfen wurde, in dem ihrer vollen Praktizjerung durch das Zerbrechen des alten Dogmas nichts mehr im Wege gestanden wäre. Die Gründe, daß die Sehnennaht von berufenster Seite, dem Präsidenten der Akademie der Chirurgie in Paris, Gilles Bertrand Pibrac (1693-1771) ${ }^{131}$ und August

127 Disc. ad Sect. I; Observ. II sect. III.

${ }^{128}$ Bonon. scient. et art. inst. comment. t. II, Bononiae 1745, p. I. S. 189-96; im Auszuge bei PaUl (vgl. Anmerkung 115) 64. ff.

${ }^{129}$ Zur Technik des Verfahrens J. L. PETIt (zitiert Anmerkung 62) 2, 289 ff., sowie A. Lours im Discours préliminaire des ersten Bandes, $21 \mathrm{ff}$.

130 Mém. 2,292.

131 G. B. Pibrac, Mémoires sur l'abus des sutures, Mém. de l'acad. roy. de chirurgie, T. III, Paris 1758, S. $424 \mathrm{ff}$. 
GоттLов RichteR in Göttingen ${ }^{132}$, verworfen wurde, waren andere: Man hatte nämlich schlechte Erfahrungen mit ihr gemacht, indem sie einerseits bei der noch unvollkommenen Nahttechnik immer wieder ausriß und anderseits sehr häufig Sekundärinfektionen zur Folge hatte.

Die Bedeutung der Regenerationsversuche der Haller-Schule scheint mir in anderer Richtung zu liegen, nämlich darin, daß in der einseitigen Betonung der Naturheilkraft das energisch und experimentell bestätigt wurde, was bisher nur einige fortschrittliche Chirurgen wußten und eigentlich nur ein einziger, Jean Louis Petit, mit seinem Stiefel auch planmäßig kurativ ausgenützt hatte: die im Sehnengewebe selbst liegenden regenerativen Kräfte ${ }^{133}$. Klar hatte sie Petit in seinem Traité des maladies des os $(2,295)$ als den einen tragenden Pfeiler seiner neuen Methode bezeichnet, indem er feststellte: «L'art et la nature ont travaillé de concert à la réunion de ces tendons rompus. L'art y étoit absolument nécessaire, soit pour rapprocher leurs bouts éloignés, soit pour les maintenir rapprochées pendant que la nature travailleroit à leur réunion.» Indem nun Haller und seine Schüler diesen wichtigen Heilungsfaktor, die Heilkraft der Natur, mit allem Nachdruck auf dem Gebiete der Sehnenverletzungen herausstellten, ist es ihnen nicht nur gelungen, auch im Regenerativen eine fundamentale Eigenschaft des lebendigen Gewebes experimentell zu erfassen; in der allzu optimistischen Weise, in der sie es taten, haben sie doch gleichzeitig auch den anfänglich von Nicolas ANdry (1658-1742) und anderen so sehr bezweifelten Heilungserfolg Petits im Falle Cochoix ${ }^{134}$ nachträglich die experimentelle Bestätigung geschaffen. Jetzt konnte sich die Bandagenbehandlung der Rupturen und Verletzungen der Achillessehne in der Chirurgie der zweiten Jahrhunderthälfte mächtig entfalten. Sie wurde in ihren verschiedenen Modifikationen durch Alexander Monro (1697-1767), Hugues Ravaton, Jakob Georg Adam Wardenburg und den Richter-Schüler Schneider die Methode der Wahl ${ }^{135}$ und ist es eigentlich solange geblieben,

132 A. G. Richter (zitiert Anmerkung 125) 7, 255.

133 Eingehend wurden sie in den experimentellen Arbeiten EnderLens, Über Sehnenregeneration, Arch. klin. Chir. 46 (1893) 563-99, und R. SEgGELs, Histologische Untersuchungen über die Heilung von Sehnenwunden, Bruns Beitr. klin. Chir. 37 (1903) $342-418$, studiert. Dort auch die ältere Literatur aus dem 19. Jahrhundert.

134 Über diesen Streit berichtet ausführlich A. Lours im Discours préliminaire (vgl. Anmerkung 62).

135 Diese Modifikationen ausführlich dargestellt bei A. G. Richter, a.a. O., S. 255 ff.; vgl. auch G. Fischer, Chirurgie vor 100 Jahren, Leipzig 1876, S. 441f. 
bis unter dem Schutze der Anti- bzw. Asepsis die große Zeit der Sehnennaht anbrach.

So hat in der mannigfachsten Weise die Hallersche Lehre von der Insensibilität der Achillessehne die aufgeklärten Geister unter den Chirurgen Europas befruchtet und zu neuer Aktivität auf einem bisher als tabu erklärten Gebiet der Wundchirurgie angespornt. Ja, selbst für die offene Achillotomie bei der Behandlung des angeborenen Klumpfußes wären die theoretischen Voraussetzungen geschaffen gewesen. Wenn sie indes nicht öfter als dreimal gewagt wurde ${ }^{136}$, 1784 von dem Lauterbacher Physicus Moriz Gerhard Thilenius (1745-1803) und dem Wundarzt Lorenz ${ }^{137}$, 1806 von Johann Friedrich Sartorius und 1809 von Christian FriedRIGH Michaelis (1754-1814) ${ }^{138}$, so deshalb, weil man einerseits, wie wir von Karl Kaspar Siebold ${ }^{139}$ wissen, Brand, Beinfraß und Tod als sekundäre Folgen scheute. Anderseits aber lebte die alte und ganz ursprüngliche Angst vor den saevissima mala der Sehnenwunden in der breiten Masse der Landbader und Feldscherer weiter fort. Noch im Krieg von $1794^{140}$ fürchteten diese nach einer Halbzerschneidung der Sehne das sofortige Auftreten von «convulsivischen Zuckungen» und «tetanischen Zufällen» so, als ob es keinen Haller und keine aufgeklärten Chirurgen in Bologna, Göttingen, Kopenhagen und Paris gegeben hätte.

136 B. Valentin, Aus der Geschichte der Orthopädie, in Handbuch der Orthopädie, 1. Band, Stuttgart 1957, S. $10 \mathrm{ff}$.

137 M. G. ThiLenius, Medicinische und chirurgische Bemerkungen, Frankfurt am Main 1789 , S. $335 \mathrm{f}$.

138 Сн. F. Michaelis, Über die Schwächung der Sehnen durch Einschneidung als einem Mittel bei manchen Gliederverunstaltungen, J. pract. Heilkde., herausgegeben von C. W. Hufeland und K. Himly, 6. Band, Jg. 1811, 5. St., S. 3 ff.

139 K. K. Siebold, Chirurgisches Tagebuch, Nürnberg 1792, S. 157.

140 Сн. F. Michaelis, a. a. O., S. 8. 